$5 \mathrm{GHz}$ band $\mathrm{n} 79$ wideband microacoustic filter using thin lithium niobate membrane

P. J. Turner, B. Garcia, V. Yantchev, G. Dyer, S. Yandrapalli, L. G. Villanueva, R. B. Hammond, V. Plessky

This article appeared in P. J. Turner et al., Electron. Lett., 55: 942-944 and may be found at https://doi.org/10.1049/el.2019.1658 


\section{$5 \mathrm{GHz}$ Band $\mathrm{n} 79$ wideband microacoustic filter using thin lithium niobate membrane}

P.J. Turner ${ }^{\bowtie}$, B. Garcia, V. Yantchev, G. Dyer, S. Yandrapalli, L.G. Villanueva, R.B. Hammond and V. Plessky

Microacoustic resonators made on suspended continuous membranes of $\mathrm{LiNbO}_{3}$ were recently shown to have very strong coupling and low losses at $>5 \mathrm{GHz}$, suitable for high-performance filter design. Employing these simple resonator structures, the authors have designed, fabricated, and measured a $4.7 \mathrm{GHz}$ bandpass laddertype filter having $1 \mathrm{~dB}$ mid-band loss and $600 \mathrm{MHz}$ bandwidth to address the $5 \mathrm{G}$ Band $\mathrm{n} 79$ requirements. The filter is fabricated on a monolithic substrate using standard i-line optical lithography and standard semiconductor processing methods for membrane release, starting with commercially available ion-sliced wafers having $400 \mathrm{~nm}$ thickness crystalline $\mathrm{LiNbO}_{3}$ layers. The filter is well-matched to a $50 \Omega$ network and does not require external matching elements. Through accurate resonator engineering using our finite element method software filter design environment, the passband is spurious-free, and the filter provides better-than $30 \mathrm{~dB}$ rejection to the adjacent WiFi frequencies. This filter demonstrates the performance and scalable technology required for high-volume manufacturing of microacoustic filters $>3.5 \mathrm{GHz}$.

Introduction: Mobile handsets rely on miniaturised high-performance radio frequency (RF) filters to implement their increasingly complex architectures, with a recent drive from new $5 \mathrm{G}$ standards to high frequencies well $>3 \mathrm{GHz}$ and wide bandwidths $>10 \%$. This presents strong challenges for incumbent $\mathrm{LiTaO}_{3} / \mathrm{LiNbO}_{3}$-based surface acoustic wave (SAW) and AlN-based bulk acoustic wave (BAW) technologies which are generally limited by lower acoustic coupling, around 3\% bandwidth, and the increasingly smaller dimensional requirements for high frequencies [1]. LTCC filters can support wide bands but require larger form factors, have a higher loss, and lack the steep rejection enabled by high-Q acoustic resonators [2].

To address this need, we have recently demonstrated laterally excited shear mode BAW resonators (XBARs) that have low losses and an extremely wide relative bandwidth of $11 \%$ at $4.8 \mathrm{GHz}$ [3]. XBARs are formed with a relatively simple structure involving a metalised interdigitated electrode (IDE) system, but with small metallisation ratio. The electrodes create predominantly horizontal electric fields which generate the half-wavelength bulk shear wave A1 resonance in the thin suspended $\mathrm{LiNbO}_{3}$ membrane. The maximum acoustic amplitude is located in the free membrane area, between said electrodes. Due to the fundamentally different acoustic mode of the XBAR, the design trade-offs are very different from conventional microacoustic resonators. In SAW, the metallic IDT electrode pitch is intimately tied to the resonator frequency, and in both SAW and BAW devices the metal thickness strongly impacts the resonator frequency and quality factor. To produce a SAW resonator operating at $5.2 \mathrm{GHz}$ comparable to our XBAR resonator, linewidths close to $0.2 \mu \mathrm{m}$ would be required. For XBAR resonators, the frequency is determined primarily by the piezoelectric plate thickness. The IDE metal thickness, as well as width, is a secondary consideration as are the line spacings which span $3-5 \mu \mathrm{m}$ and are easily produced with optical lithography. To realise the spurious free low-loss design of a monolithic bandpass ladder filter, a coating of $\mathrm{SiO}_{2}$ material on the shunt resonators is used to tune the operating frequency.

Filter design: Highly accurate finite element method (FEM) simulations using Resonant's ISN(C) for both the microacoustic resonators $[4,5]$ and electromagnetic (EM) circuit allow rapid design cycles exploring a large parameter space. The design procedure begins with the synthesis of a lumped-element model for the resonators in the absence of any EM interconnect circuit simulation, as in Fig. 1, with the number of ladder sections determining the ultimate filter rejection level. Butterworth van Dyke (BVD) resonator parameters for the initial approximate prototype design are provided in Table 1, demonstrating the useful element values appropriate for RF filter design that can be achieved with this technology. These parameters were used for the initial BVD design of the filter shown in Fig. 2. At the following design steps, the filter is realised and optimised with FEM models for the XBARs and EM interconnects.

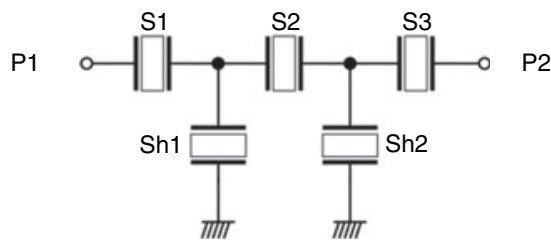

Fig. 1 Schematic diagram of five-resonator, two-port prototype ladder filter using three series resonators and two shunt (parallel branch) resonators

Table 1: Equivalent BVD circuit parameters for the resonators used in the prototype filter design

\begin{tabular}{|c|c|c|c|c|}
\hline Resonator & $\mathrm{C} 0, \mathrm{pF}$ & $\mathrm{Cm}, \mathrm{pF}$ & $\mathrm{Lm}, \mathrm{nH}$ & $\mathrm{Fr}, \mathrm{MHz}$ \\
\hline series 1 & 0.43 & 0.113 & 9.9 & 4746 \\
\hline series 2 & 0.25 & 0.670 & 16.8 & 4717 \\
\hline series 3 & 0.61 & 0.171 & 6.7 & 4696 \\
\hline shunt 1 & 1.22 & 0.303 & 4.8 & 4165 \\
\hline shunt 2 & 1.19 & 0.298 & 4.9 & 4147 \\
\hline
\end{tabular}

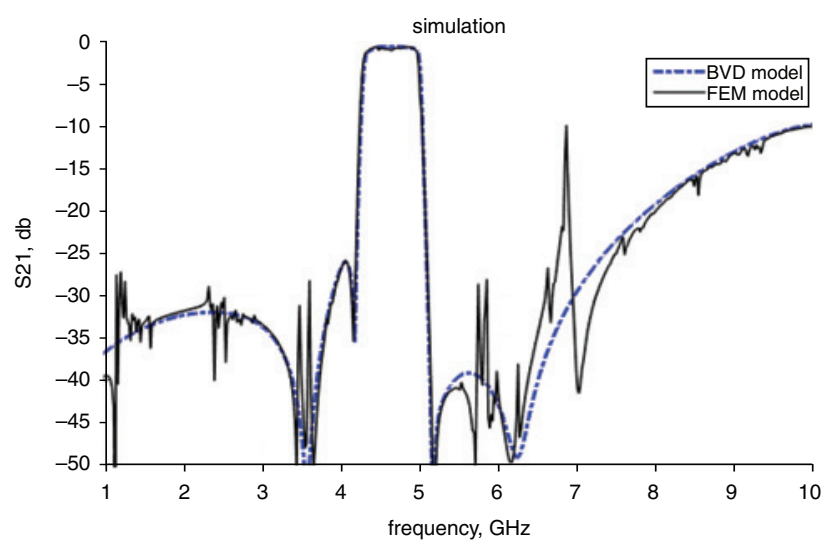

Fig. 2 Simulation of the prototype XBAR filter near the $5 G$ Band $n 79$ frequency. Accurate FEM models for the resonators and EM interconnect layout allow filter optimisation after the BVD model is realised

Realising the design with XBARs requires controlling and minimising spurious modes which must be avoided in the critical regions of the filter. Special attention must be paid to the higher frequency A1-3 ('horizontal third harmonic' resonance [3]), which has the same nature as the primary $\lambda / 2$ thickness resonance A1 and is easily excited. Sharp parasitic propagating modes, such as the lowest order A0 and S0 Lamb modes and shear SHO modes, can also be a problem. Although the resonators have moderate $Q$-factors - estimated to be around 500 - in combination with extremely high coupling we predict a record-low minimal insertion loss of $1 \mathrm{~dB}$ near $5 \mathrm{GHz}$.

Fabrication: The XBAR filter is implemented on an ion-sliced $400 \mathrm{~nm}$ thin film of $Z Y$-oriented single-crystal $\mathrm{LiNbO}_{3}$ bonded to a $250 \mu \mathrm{m}$ thick Si carrier wafer from NanoLN [6]. Fig. 3 shows a microscope image of the resulting filter. Frontside IDE lithography uses an ASML 5500 DUV stepper and liftoff process for the $\mathrm{Al}$ metal layer. A second pad metallisation using thick $\mathrm{Au}$ reduces interconnect loss. A sputtered $\mathrm{SiO}_{2}$ coating layer is deposited on the shunt resonators, defined by lithography and liftoff. Subsequent XBAR membrane release is performed with a backside $\mathrm{Si}$ deep reactive-ion etch (DRIE) process, followed by a HF etch to remove the buried $\mathrm{SiO}_{2}$ bonding layer under the membrane. A frontside etch process is also possible, with Si DRIE performed through openings in the $\mathrm{LiNbO}_{3}$ layer on the periphery of the IDE. In both cases, the released membranes are delicate and require special wafer handling. However, the membrane is solidly attached to the Si wafer on all sides and is more robust than the standard MEMS anchor attachment for suspended membranes. Following fabrication, the wafer is diced to release the filter chips.

Results: Figs. 4 and 5 present wafer-probe measured filter performance for an improved design showing over $600 \mathrm{MHz}$ of bandwidth, $1.35 \mathrm{~dB}$ minimum insertion loss, adjacent WiFi rejection approaching $35 \mathrm{~dB}$, and well-behaved wideband rejection all the way to $10 \mathrm{GHz}$. The measured passband shows the absence of strong spurious modes; 
however, small amplitude $12 \mathrm{MHz}$ passband ripples are observed caused by backside acoustic reflections from the polished Si substrate. Some similar designs, specifically engineered to increase their RF power handling ability, have been measured to exceed $31 \mathrm{dBm} C W$ input power at room temperature across the $600 \mathrm{MHz}$ band. Further power handling improvement will continue.

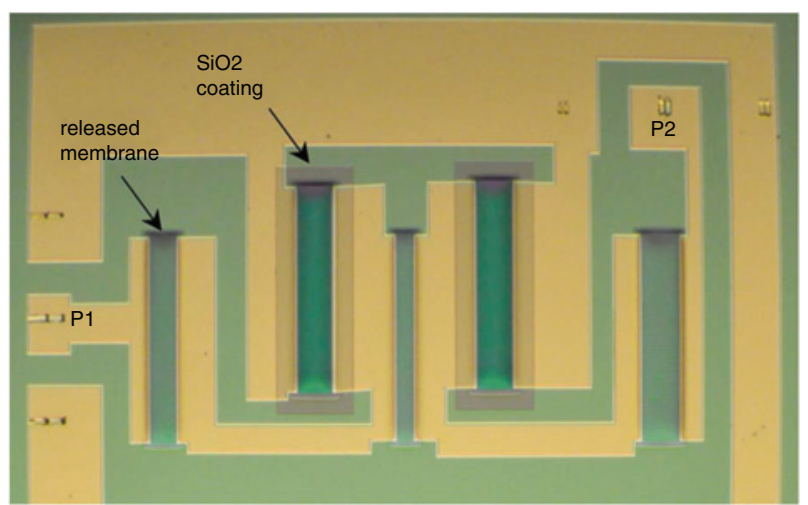

Fig. 3 Microscope image of a prototype 5-resonator XBAR ladder filter. Etch-released membranes are visible as dark areas under each resonator and $\mathrm{SiO}_{2}$ overcoat is visible as a grey coating on the shunt resonators. IDE metallisation is Al and interconnect metal has a second Au layer to reduce ohmic loss

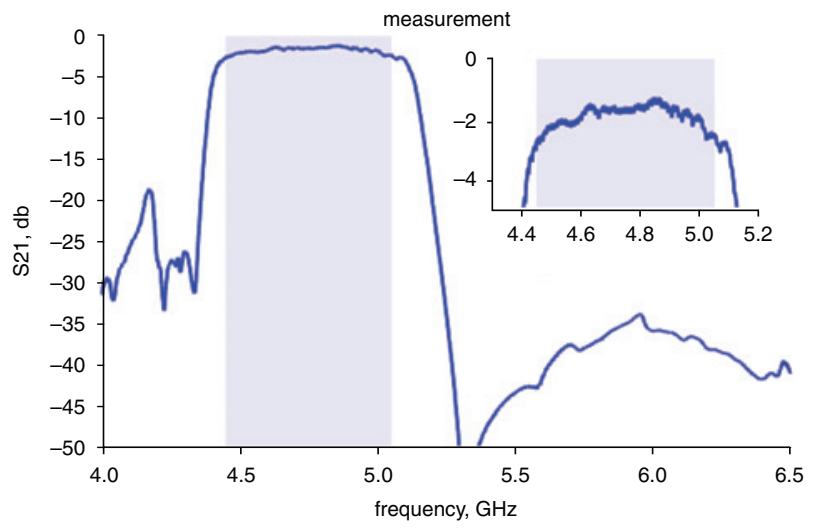

Fig. 4 Wafer probe measurements of an improved XBAR filter showing $1.35 \mathrm{~dB}$ minimum passband insertion loss with a spurious-free $600 \mathrm{MHz}$ bandwidth (shaded region) close to the $5 G$ Band $n 79$ specification. The singulated filter die size is $1.8 \times 1.4 \mathrm{~mm}^{2}$ and the part requires no matching elements to $50 \Omega$. This filter is capable of $31 \mathrm{dBm}$ input $R F C W$ power handling at room temperature

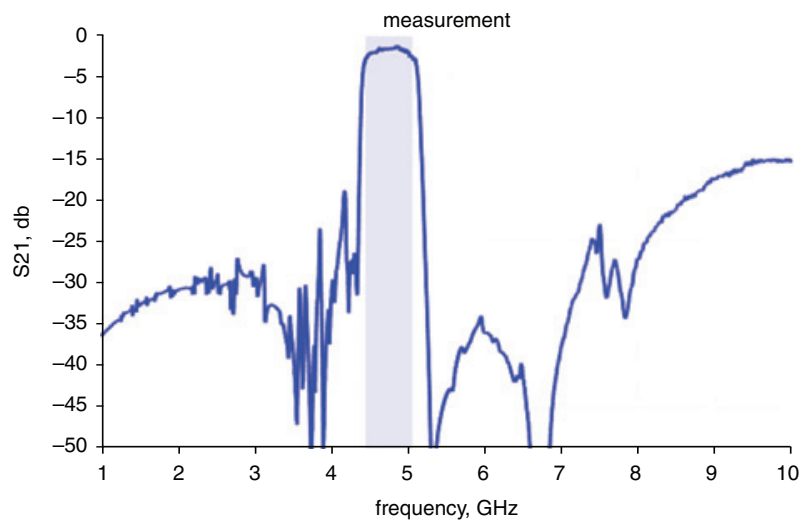

Fig. 5 Wideband wafer-probe measurements of an improved XBAR filter showing good rejection and well-behaved response from $1-10 \mathrm{GHz}$
Conclusions: We report the first measured results on a prototype ladder filter composed of laterally excited shear mode acoustic resonators (XBARs) fabricated from sub-mm thickness $\mathrm{LiNbO}_{3}$ platelets operating in the $5 \mathrm{GHz}$ frequency range, suitable for $5 \mathrm{G}$ mobile phone applications. This filter demonstrates excellent performance and confirms that a new technology using ion-sliced mono-crystalline layers of $\mathrm{LiNbO}_{3}$ opens new horizons in microacoustic filters. Although the acoustic wavelength in the XBARs is sub-micron, the electrode lithography does not need to scale well below this to produce lines on the order of $\lambda / 4$, the technologically challenging requirement that limits manufacturable SAW resonator frequencies. Furthermore, the strongly coupled shear-mode resonance used in XBARs is largely decoupled from the IDE metal, resulting in excellent performance with considerable flexibility in engineering device performance. This Band n79 filter demonstrates the performance and scalable technology required for high-volume manufacturing of microacoustic filters well above $5 \mathrm{GHz}$.

(C) The Institution of Engineering and Technology 2019

Submitted: 10 May 2019 E-first: 17 July 2019

doi: 10.1049/el.2019.1658

One or more of the Figures in this Letter are available in colour online. P.J. Turner, B. Garcia, V. Yantchev, G. Dyer and R.B. Hammond (Resonant Inc., Santa Barbara, CA, USA)

凶 E-mail: pturner@resonant.com

S. Yandrapalli and V. Plessky (Resonant Inc. and GVR Trade SA, Gorgier, Switzerland)

L.G. Villanueva (ANEMS Laboratory, EPFL, Lausanne, Switzerland)

S. Yandrapalli: Also with ANEMS Laboratory, EPFL, Lausanne, Switzerland

\section{References}

1 Ruppel, C.: 'Acoustic wave filter technology - a review', Trans. Ultrason. Ferroelectr. Freq. Control, 2017, 67, (9), pp. 1390-400

2 Mahon, S.: 'The 5G effect on RF filter technologies', Trans. Semicond. Manuf., 2017, 30, (4), pp. 494-499

3 Plessky, V., Yandrapalli, S., Turner, P.J., et al.: ' $5 \mathrm{GHz}$ laterally-excited bulk-wave resonators (XBARs) based on thin platelets of lithium niobate', Electron. Lett., 2018, 55, (2), pp. 98-100

4 Koskela, J., Maniadis, P., Willemsen, B.A., et al.: 'Hierarchical cascading in 2D FEM simulation of finite SAW devices with periodic block structure'. IEEE Int. Ultrasonics Symp., Tours, France, September 2016, pp. 1-4

5 Plessky, V., Maniadis, P., Willemsen, B.A., et al.: 'FEM modeling of an entire 5-IDT CRF/DMS filter'. IEEE Int. Ultrasonics Symp., Washington, DC, USA, September 2017, pp. 1-5

6 NanoLN: 'LNOI - LN thin film on insulator', available at http://www. nanoln.com/, accessed May 2019 